\title{
Synchronous diagnosis of a hodgkin lymphoma and a papillary carcinoma of the thyroid
}

\author{
Jamale J oseph Rizkallah, Sélim S Jambart, Georges Daniel Maalouli \\ Faculty of Medicine, Saint J oseph University, Hotel Dieu de France Hospital, Beirut, Lebanon. \\ Correspondence: Jamale J oseph Rizkallah. Address: Faculty of Medicine, Saint J oseph University, Hotel Dieu de France \\ Hospital, Beirut, Lebanon. Email: jamale87@yahoo.fr
}

Received: May 31, 2014

Accepted: July 15, 2014

Online Published: August 20, 2014

DOI : $10.5430 /$ crim.v1n2p235

URL: http://dx.doi.org/10.5430/crim.v1n2p235

\begin{abstract}
The increased incidence of thyroid cancer is probably the result of the increased detection of subclinical disease. We present the case of a 24-year-old male patient, with no pre-existing personal or family history of thyroid disease or past irradiation exposure, who was concomitantly diagnosed with a Hodgkin lymphoma and a papillary carcinoma of the thyroid in the context of B signs and the absence of thyroid disease symptoms. This case illustrates a unique and previously unreported example of two synchronous cancers of which one was fortuitously discovered. We also report the treatment approach we adopted.
\end{abstract}

\section{Keywords}

Hodgkin lymphoma, Papillary thyroid cancer

\section{I ntroduction}

There are over 30,000 new cases of thyroid cancer each year, representing approximately $2.5 \%$ of cancer diagnoses. Among these, incidentally diagnosed thyroid papillary carcinomas are increasing in incidence ${ }^{[1]}$. We present the case of a 24-year-old male patient, diagnosed with concomitant thyroid papillary carcinoma and Hodgkin lymphoma during a mediastinoscopy undertaken for thoracic lymph node biopsy. This case illustrates a previously unreported example of two synchronous cancers of which one was fortuitously discovered.

\section{Case presentation}

This is the case of a 24-year-old male patient, known asthmatic, with no other personal or family medical history and no know history of past exposure to radiation. He presented to a private clinic complaining of $10 \%$ weight loss over one month, persistent fever of $38.5^{\circ} \mathrm{C}$, night sweats and persistent non-bloody cough. No other symptoms were reported. Labs were within normal limits. The thoracic CT scan showed diffuse adenopathies of the left upper mediastinum, the Barety space, the right hilum, the aorto-pulmonary window as well as laterally to the aorta and below the carina.

A mediastinoscopy with lymph nodes biopsy was performed. The pathology of the right paratracheal adenopathy showed suspicious material considered as follicular lymphoid cells on the frozen section. The final pathology reported nodular sclerosis classical of Hodgkin lymphoma. The Hodgkin cells were positive for CD30 and CD14 on immunohisto- 
chemistry. In parallel, a cervical lesion found during the mediastinoscopy also biopsied was identified by pathology as a thyroid papillary carcinoma infiltrating the muscular and adipose tissue.

Explorations with serologic measures of calcitonin, thyroglobulin and TSH were consequently done: Calcitonin = 2.8pg/ml (< 8.5), Thyroglobulin $=3.5 \mathrm{ng} / \mathrm{ml}(0.83-68)$ and TSH $=0.6 \mathrm{mlU} / \mathrm{l}(0.3-4)$. A cervical ultrasound showed a homogenous thyroid gland with an extension from the isthmus, measuring $8.4 \mathrm{~cm}$, infiltrating the capsula and harboring microcalcifications.

A whole body PET-Scan revealed small active focus of the right isthmus of the thyroid gland, probably of neoplastic nature, as well as an active nodal disease at the neck region and the thorax with large conglomerates of matted nodes seen in the peri-carinal region. This was considered to be secondary to a lymphatic spread of thyroid carcinoma and/or secondary to the lymphomatous disease.

The patient underwent treatment for Hodgkin lymphoma by 4 cycles of ABVD (doxorubicin, bleomycin, vinblastine and dacarbazine) and 20 Gy of external beam radiotherapy (EBRT). The follow up PET-Scan didn't show any abnormal activity. As for the thyroid cancer, a complete thyroidectomy was undertaken 3 months later followed by radioiodine treatment with $100 \mathrm{mCu}$ of ${ }^{131} \mathrm{I}$ a couple of weeks later and was prescribed thyroid hormones as replacement therapy. The patient was discharged and advised for close follow up.

\section{Discussion}

In this article, investigations for a case of Hodgkin lymphoma allowed an incidental diagnosis of papillary thyroid cancer. The patient presented in the context of fever, weight loss and night sweats. These typical B signs suggested the diagnosis of lymphoma, particularly Hodgkin's lymphoma because of the young age of the patient. On thoracic lymph node biopsy, Hodgkin's lymphoma was confirmed and an incidental thyroid papillary carcinoma was fortuitously discovered. The dilemma was raised about the indications for treatment of this asymptomatic incidental thyroid carcinoma, the effect of the therapeutical time interval (TTI) before treating the thyroid and the use of chemotherapy of the Hodgkin's lymphoma treatment panel that might be of effect to the thyroid carcinoma.

According to our review of literature, differentiated thyroid cancer (DTC), predominantly papillary (85\% of cases), is the most common thyroid cancer, with a documented increased incidence in the last decades ${ }^{[2]}$. The incidence of papillary cancer increased by 2.9-fold, from 2.7 to 7.7 per 100,000. This is predominantly due to the increased detection of small papillary cancers. This, combined with the known existence of a substantial reservoir of subclinical cancer and a stable overall mortality, suggest that the increasing incidence reflects increased detection of subclinical disease and not an increase in the occurrence of thyroid cancer ${ }^{[2]}$. As a matter of fact, most patients with papillary cancer do not die of their disease. The likelihood of thyroid cancer death is increased by age $\geqslant 40$ years, tumor size $\geqslant 1.5 \mathrm{~cm}$, local tumor invasion, regional lymph-node metastases, and a delay in therapy of more than a year; it is reduced by female sex, complete resection, and adjuvant use of ${ }^{131} \mathrm{I}$ and thyroid hormone therapy ${ }^{[3]}$. Based upon this decreased morbidity and mortality, the treatment of the Hodgkin's lymphoma was initiated first especially that the TTI for the treatment of thyroid papillary carcinomas does not affect morbidity or mortality ${ }^{[4]}$. The patient was diagnosed with Hodgkin's lymphoma stage IB and was treated according to the NCCN guidelines with 4 cycles of ABVD and 20 Gy of EBRT. Explorations demonstrated at the end of treatment complete remission of the lymphoma.

As for the treatment recommendations for thyroid papillary carcinoma, surgery is the primary mode of therapy. Total thyroidectomy is recommended if the primary tumor is at least $1-2 \mathrm{~cm}$ in diameter, or if extrathyroidal extension or metastases are present. The rationale for radioiodine use in the adjuvant setting includes ablation of residual macroscopic and microscopic thyroid tissue and treatment of a known metastatic thyroid cancer ${ }^{[5]}$, or in primary tumor $>4 \mathrm{~cm}$ even in the absence of high risk features such as vascular invasion, more aggressive histologic subtypes, such as tall cell, columnar cell, insular, or poorly differentiated histologies when the combination of age, tumor size, lymph node status, and 
individual histology predicts an intermediate to high risk of recurrence or death from thyroid cancer ${ }^{[6]}$. After initial thyroidectomy, whether or not radioiodine therapy is administered, levothyroxine therapy is required for all patients to minimize potential TSH stimulation of tumor growth and to prevent hypothyroidism. As for the treatment of papillary carcinoma incidentally diagnosed, a complete resection with post- thyroidectomy treatment if needed is required. Another alternative option for incidental multifocal microcarcinomas would be a subtotal thyroidectomy followed by radioiodine use without total thyroidectomy and lymphadenectomy ${ }^{[7]}$. The patient was approached according to this review and treated as such.

Chemotherapy was reported to be used in trials for thyroid cancers, particularly anaplastic thyroid carcinomas. The main drugs studied were cisplatin, doxorubicin, etoposide and peplomycin. Doxorubicin is a medication of the Hodgkin's chemotherapy protocols but was never studied in the thyroid papillary carcinoma setting. In our case, we did not observe any response of the thyroid disease to the chemotherapy administered. Undergoing clinical studies are studying BRAF targeted therapy, approved now in metastatic melanoma, as well as tyrosine kinase inhibitors such as sorafenib and vandetabib, but the anticancerous effect has not been demonstrated yet ${ }^{[8]}$.

To our knowledge, no relation was described between Hodgkin's lymphoma and papillary thyroid carcinoma other than an increased risk of thyroid cancer after radiotherapy for Hodgkin's disease which is not the case of our patient.

\section{Conclusion}

We report the case of a 24 year old male patient who was incidentally diagnosed with thyroid papillary carcinoma during the work up for a suspected Hodgkin's lymphoma. We couldn't find any relation between these two separate entities other than an increased risk of thyroid cancer after radiotherapy for Hodgkin's disease. Therapy was individualized, aimed at the more aggressive tumor, but state of the art optimal treatment was delivered for both diseases. The chemotherapy used for Hodgkin's disease was of no effect on the papillary cancer.

\section{Conflict of interest}

There are no financial nor non-financial competing interests regarding this case report.

\section{References}

[1] Ji Lu. Secondary Lymphoma Involving Metastatic Follicular Thyroid Carcinoma to the Skull: A Unique Example of Tumor-to-Tumor Metastasis, Head Neck Pathol. 2008 Sep; 2(3): 209-212. http://dx.doi.org/10.1007/s12105-008-0051-8

[2] Davies L, Welch HG. Increasing incidence of thyroid cancer in the United States, 1973-2002. JAMA. 2006; 295 (18): 2164. http://dx.doi.org/10.1001/jama.295.18.2164

[3] Mazzaferri EL, Jhiang SM. Long-term impact of initial surgical and medical therapy on papillary and follicular thyroid cancer. Am J Med. 1994; 97(5): 418. http://dx.doi.org/10.1016/0002-9343(94)90321-2

[4] Dionigi G, Tanda M, Piantanida E, Boni L, Rovera F, Dionigi R, et al. Time interval in diagnosis and treatment of papillary throid cancer: a descriptive, retrospective study. 2009 Apr; 197(4): 434-8.

[5] Martin Schlumberger, et al. Strategies of Radioiodine Ablation in Patients with Low-Risk Thyroid Cancer in The new England journal of medicine. 2012 May; 366 (18): 1663-1673.

[6] Cooper DS, Doherty GM, Haugen BR, Hauger BR, Kloos RT, Lee SL, et al. Revised American Thyroid Association management guidelines for patients with thyroid nodules and differentiated thyroid cancer. American Thyroid Association (ATA) Guidelines Taskforce on Thyroid Nodules and Differentiated Thyroid Cancer, Thyroid. 2009; 19(11): 1167. http://dx.doi.org/10.1089/thy.2009.0110

[7] Dietlein M, Luyken WA, Schicha H, Larena-Avellaneda A. Incidental multifocal papillary microcarcinomas of the thyroid: Is subtotal thryodiectomy combined with radioiodine ablation enough. 2005 Jan; 26(1): 3-8.

[8] Deshpande, Hari A View Author Profile; Gettinger, Scott N View Author Profile; Sosa, Julie A, Targeted therapy for thyroid cancer: An updated review of investigational agents." Current opinion in investigational drugs (London, England: 2000) 2000; 11 (6): 661-8. 